MSC 65J15

DOI: $10.14529 /$ jcem150105

\title{
NUMERICAL MODELING OF QUASI-STEADY PROCESS IN CONDUCTING NONDISPERSIVE MEDIUM WITH RELAXATION
}

E. A. Bogatyreva, South Ural State University, Chelyabinsk, Russian Federation, bogatyreva-ea@susu.ac.ru

Sufficient conditions of existence and uniqueness of weak generalized solution to the Dirichlet-Cauchy problem for equation modeling a quasi-steady process in conducting nondispersive medium with relaxation are obtained. The main equation of the model is considered as a representative of the class of quasi-linear equations of Sobolev type. It enables to prove a solvability of the Dirichlet-Cauchy problem in a weak generalized meaning by methods developed for this class of equations. In suitable functional spaces the DirichletCauchy problem is reduced to the Cauchy problem for abstract quasi-linear operator differential equation of the special form. Algorithm of numerical solution to the DirichletCauchy problem based on the Galerkin method is developed. Results of computational experiment are provided.

Keywords: Galerkin method, quasi-linear Sobolev type equation, weak generalized solution, numerical modeling.

\section{Introduction}

Assume that $\Omega \subset \mathbb{R}^{n}, n \geq 2$ is a bounded region with boundary of class $C^{\infty}$. Consider the Dirichlet-Cauchy problem

$$
\begin{gathered}
u(x, t)=0,(x, t) \in \partial \Omega \times(0, \tau), \\
u(x, 0)=u_{0}(x), x \in \Omega,
\end{gathered}
$$

for the equation

$$
(\Delta u-\Phi(u))_{t}=\Phi(u)
$$

in cylinder $\Omega \times T, T \in \mathbb{R}$.

This problem arises during a research of quasi-steady processes in conducting nondispersive media [1]. Unknown function $u$ corresponds to the electric field potential. Function $\Phi(u) \equiv|u|^{p-2} u, p>2$ is monotonely increasing and smooth. Problem (1) - (3) was considered earlier in the [2], global solvability in strong generalized meaning was established under some conditions. We consider the equation (3) as a representative of the class of quasi-linear equations of Sobolev type. It enables us to prove a solvability of problem (1) - (3) in a weak generalized meaning by methods developed for this class of equations.

In suitable functional spaces we reduce problem (1) - (3) to the Cauchy problem

$$
u(0)=u_{0}
$$

for abstract operator differential equation of the form

$$
\frac{d}{d t}(L(u))+M(u)=0
$$


where $L(u)=A u+\lambda M(u), \lambda \in \mathbb{R}_{+}$. Equation (5) is a quasi-linear Sobolev type equation. Nonsolvable in relation to high derivative equations attract the attention of many researchers [3] - [7]. Problem (4), (5) was considered in the [8], conditions of existence and uniqueness of the weak generalized solution were developed.

The article contains two parts. Reduction of problem (1) - (3) to the abstract problem is developed, and the theorem of existence and uniqueness of weak generalized solution to problem (1) - (3) is provided in the first part. Results of the computational experiment based on the theoretical results are provided in the second part.

\section{Solvability}

Introduce some definitions and assumptions necessary for further consideration.

Assume that $\mathfrak{H}=(\mathfrak{H},\langle\cdot, \cdot\rangle)$ is a real Hilbert space identified with its dual and equipped with dual pairs of reflexive Banach spaces $\mathfrak{U} \equiv(\mathfrak{U},\|\cdot\|), \mathfrak{U}^{*} \equiv\left(\mathfrak{U},\|\cdot\|_{*}\right), \mathfrak{F} \equiv(\mathfrak{F},\|\cdot\|)$ and $\mathfrak{F}^{*} \equiv\left(\mathfrak{F},\|\cdot\|_{*}\right)$ such that we have a continuous dense embedding

$$
\mathfrak{U} \hookrightarrow \mathfrak{F} \hookrightarrow \mathfrak{H} \hookrightarrow \mathfrak{F}^{*} \hookrightarrow \mathfrak{U}^{*}
$$

Definition 1. Refer as a weak generalized solution to the Cauchy problem (4), (5) to a function $u(t) \in L_{\infty}(0, \tau, \mathfrak{U})$, with $\frac{d u}{d t} \in L_{2}(0, \tau, \mathfrak{U})$, satisfying

$$
\begin{gathered}
\int_{0}^{\tau}\left(\frac{d}{d t}\langle L(u), w\rangle+\langle M(u), w\rangle\right) \varphi(t) d t=0, \\
u(0)=u_{0}, \forall w \in \mathfrak{U}, \forall \varphi \in L_{2}(0, \tau) .
\end{gathered}
$$

Condition 1. $\exists F(s) \geq 0$ for almost all $s \in[0, \infty)$, such that $F \in C[0, \infty)$ possibly after a change on a negligible set, and for almost all $s_{0} \in[0, \infty)$, for any $u=u\left(s_{0}\right), v=v\left(s_{0}\right) \in \mathfrak{U}$ condition

$$
\|M(u)-M(v)\|_{*} \leq F\left(s_{0}\right)\|u-v\| .
$$

is satisfied.

Condition 2. $\exists C^{M}>0$, and $\exists p \geq 2$ such that $\|M(u)\|_{*} \leq C^{M}\|u\|^{p-1} \forall u \in \mathfrak{U}$ and $\langle M(u), u\rangle \geq 0$.

Assume that $M \in C^{r+1}\left(\mathfrak{F} ; \mathfrak{F}^{*}\right), r \in \mathbb{N}$, is $s$-monotonous, homogeneous of degree $k$ and satisfies to conditions 1 and 2, and, furthermore, Fréchet derivative of the operator $M$ is symmetric, and the operator $A \in \mathfrak{L}\left(\mathfrak{U} ; \mathfrak{U}^{*}\right)$ is symmetric and positive definite.

Theorem 1. Suppose that the unique local solution to problem (4), (5) exists for some interval $\left(-\tau_{0}, \tau_{0}\right), \tau_{0} \in \mathbb{R}_{+}$. Then there exists a unique weak generalized solution to problem (4), (5).

Proof.

The proof is completely similar to one provided in [8], except the requirement of $p$ coercivity of operator $M$ is replaced by the weaker condition 2 . 
To reduce problem $(1)$ - (3) to problem $(4),(5)$ assume $\mathfrak{H}=L_{2}(\Omega), \mathfrak{U}=\stackrel{0}{W}^{1}(\Omega)$ and $\mathfrak{F}=L_{p}(\Omega)$. Note that we have continuous dense embeddings (6) because of the Sobolev embedding theorem [9, p. 53].

Define operators $A$ and $M$ as follows:

$$
\begin{gathered}
\langle A u, v\rangle=\int_{\Omega} \nabla u \nabla v d x, u, v \in \mathfrak{U}, \\
\langle M(u), v\rangle=\int_{\Omega}|u|^{p-2} u v d x, \quad u, v \in \mathfrak{F} .
\end{gathered}
$$

Lemma 1. Operator $A: \mathfrak{U} \rightarrow \mathfrak{U}^{*}$ is linear, positive definite, symmetric and continuous.

Lemma 2. Operator $M \in C^{2}\left(\mathfrak{F} ; \mathfrak{F}^{*}\right)$, is s-monotonous, homogeneous of degree $k$ and satisfies to conditions 1 and 2 , and Fréchet derivative of the operator $M$ is symmetric.

Proof.

First show the effect of the operator $M: \mathfrak{F} \rightarrow \mathfrak{F}^{*}$. Because of the Hölder's inequality and embeddings (6) we have

$$
|\langle M(u), v\rangle| \leq \int_{\Omega}|u|^{p-1}|v| d x \leq\|u\|_{L_{p}}^{p-1}\|v\|_{L_{p}} .
$$

therefore

$$
\|M(u)\|_{*}=\sup _{\|v\|=1}|\langle M(u), v\rangle| \leq C\|u\|_{L_{p}}^{p-1}
$$

i.e, operator $M: \mathfrak{F} \rightarrow \mathfrak{F}^{*}$ actually. Moreover, operator $M$ satisfies to condition 2 because of (7) and

$$
\langle M(u), u\rangle=\int_{\Omega}|u|^{p} d x \geq 0 .
$$

It is evident that operator $M$ is homogeneous of degree $p-1$.

Further, develop the Fréchet derivative $M_{u}^{\prime}$ of the operator $M$. At the point $u \in \mathfrak{F}$ it is defined by formula

$$
\left\langle M_{u}^{\prime} v, w\right\rangle=(p-1) \int_{\Omega}|u|^{p-2} v w d x, u, v, w \in \mathfrak{F}
$$

and is symmetric. Because of the Hölder's inequality and embeddings (6) we have

$$
\left|\left\langle M_{u}^{\prime} v, w\right\rangle\right|=(p-1) \int_{\Omega}|u|^{p-2}|v w| d x \leq(p-1)\|u\|_{L_{p}}^{p-2}\|v\|_{L_{p}}\|w\|_{L_{p}}
$$

operator $M_{u}^{\prime} \in \mathfrak{L}\left(\mathfrak{F} ; \mathfrak{F}^{*}\right)$ for all $u \in \mathfrak{F}$. Prove the $s$-monotonity of operator $M$ :

$$
\left\langle M_{u}^{\prime} v, v\right\rangle=(p-1) \int_{\Omega}|u|^{p-2} v^{2} d x>0, u, v \in \mathfrak{F} \backslash\{0\} .
$$


Demonstrate the inclusion $M \in C^{2}\left(\mathfrak{F} ; \mathfrak{F}^{*}\right)$ :

$$
\left|\left\langle M_{u}^{\prime \prime}(v, w), z\right\rangle\right| \leq(p-1)(p-2) \alpha\|u\|_{L_{p}}^{p-3}\|v\|_{L_{p}}\|w\|_{L_{p}}\|z\|_{L_{p}} .
$$

Finally, prove that operator $M$ satisfies to condition 1 .

There exists a nonnegative continuous function $g: \mathbb{R}^{2} \rightarrow \mathbb{R}$ such that

$$
\left.|| u\right|^{p-2} u-|v|^{p-2} v|\leq g(u, v)| u-v \mid
$$

for all $u, v \in \mathbb{R}$. It is easy to show that, for instance, the function

$$
g(u, v)=\left\{\begin{array}{l}
\frac{\left.|| u\right|^{p-2} u-|v|^{p-2} v \mid}{|u-v|} \text { for } u \neq v \\
(p-2)|u|^{p-1} \text { for } u=v
\end{array}\right.
$$

satisfies these conditions. By (8), for all real-valued functions $u=u(x, s), v=v(x, s) \in \mathfrak{U}$

$$
\left.|| u\right|^{p-2} u-|v|^{p-2} v|\leq f(x, s)| u-v \mid
$$

almost everywhere, where $f(x, s)=g(u(x, s), v(x, s))$. It follows from (9) that

$$
\sup _{\|w\|=1} \alpha \int_{\Omega}\left\|\left.u\right|^{p-2} u-|v|^{p-2} v\right\| w\left|d x \leq \sup _{\|w\|=1} \alpha \int_{\Omega} f(x, s)\right| u-v \| w \mid d x .
$$

In the left-hand side we have

$$
\begin{gathered}
\left.\sup _{\|w\|=1} \alpha \int_{\Omega}|| u\right|^{p-2} u-|v|^{p-2} v \| w\left|d x \geq \sup _{\|w\|=1} \alpha\right| \int_{\Omega}|u|^{p-2} u w d x-\int_{\Omega}|v|^{p-2} v w d x \mid= \\
=\sup _{\|w\|=1}|\langle M(u)-M(v), w\rangle|=\|M(u)-M(v)\|_{*} .
\end{gathered}
$$

In the right-hand side we have

$$
\begin{gathered}
\sup _{\|w\|=1} \int_{\Omega} f(x, s)\left|u-v\left\|w \left|d x=\sup _{\|w\|=1}\langle f(x, s)|u-v|,|w|\rangle \leq \sup _{\|w\|=1}\|f(x, s)|u-v|\|_{L_{2}}\|w\|_{L_{2}} \leq\right.\right.\right. \\
\leq A\|f(x, s)|u-v|\|_{L_{2}} \leq C\|f(x, s)\|_{L_{2}}\|u-v\|_{L_{2}} \leq F(s)\|u-v\|_{L_{p}} .
\end{gathered}
$$

Hence,

$$
\|M(u)-M(v)\|_{*} \leq F(s)\|u-v\| .
$$

Existence of the unique local solution to the problem (1) - (3) was proved in [9] for any initial conditions assuming that $p>2$.

Hence, the next theorem holds.

Theorem 2. Assume that $2<p \leq$ frac2nn -2 , then for any $u_{0} \in{\stackrel{0}{W_{2}^{1}}}^{1}(\Omega)$ and for any $\tau \in \mathbb{R}_{+}$there exists a unique weak generalized solution to problem (1) - (3). 


\section{Computational Experiment}

Algorithm of numerical solution to problem (1) - (3) and modeling a quasisteady process in conducting medium with relaxation was developed and implemented in Maple 15.0 environment basing on the theoretical results. The developed program allows us:

1. To specify initial condition $u_{0}(r, \phi)$, radius $R$ of circle in which the problem is solved, number $N$ of Galerkin approximations.

2. To find an approximate solution to the Dirichlet-Cauchy in the circle with initial conditions specified.

3. To show the graph of the approximate solution on the display.

For example, let us find a numerical solution to problem (1) - (3) in the circle of radius $R=1$ with conditions: $u_{0}=1-r^{2}, \Phi(u)=u^{3}(r, \phi, t)$. Initial and boundary conditions are symmetric (independent of the variable $\phi)$. Provide the problem (1) - (3) with formulated conditions:

$$
\left\{\begin{array}{c}
\left(\frac{1}{r}\left(r(u(r, t))_{r}\right)_{r}\right)_{t}-\left(u^{3}(r, t)\right)_{t}=u^{3}(r, t), \\
u(r, 0)=1-r^{2} \\
u(1, t)=0, \text { при } \phi \in[0 ; 2 \pi] .
\end{array}\right.
$$

Define the set of eigenfunctions of homogeneous Dirichlet problem for Laplace operator in the circle of radius $R=1$ orthonormal with scalar product in space $L_{2}(\Omega)$ as $\left\{\Phi_{k}\right\}$. We represent an unknown function in the form of Galerkin summ:

$$
u(r, t)=\sum_{i=k}^{\infty} u_{k}(t) \Phi_{k}(r) .
$$

Let us find the approximate solution with 2 Galerkin approximations in the summ:

$$
u(r, t)=\frac{\sqrt{2}}{J_{1}\left(\mu_{1}^{0}\right)} J_{0}\left(r \mu_{1}^{0}\right) u_{1}(t)+\frac{\sqrt{2}}{J_{1}\left(\mu_{2}^{0}\right)} J_{0}\left(r \mu_{2}^{0}\right) u_{2}(t),
$$

where $\mu_{i}^{(k)}$ is a $i$-th zero of $J_{k}$ function. Substitute this representation to the equation. Taking the scalar product with eigenfunctions of Laplace operator, we get differential system for the coefficients $u_{1}(t)$ and $u_{2}(t)$. Solving this system numerically, we get the approximate solution to the problem (10). Graphs of the approximate solution at various time points $(t=0, t=5, t=10, t=50)$ are shown in Figure 1.

\section{References}

1. Korpusov M.O., Pletner Yu.D., Sveshnikov A.G. On Quasi-Steady Processes in Conducting Nondispersive Media. Computational Mathematics and Mathematical Physics, 2000, vol. 40, no 8, pp. 1188-1199.

2. Korpusov M.O. Blowup of the Solution to a Pseudoparabolic Equation with the Time Derivative of a Nonlinear Elliptic Operator Computational Mathematics and Mathematical Physics, 2002, vol. 42, no 12, pp. 1717-1724. 

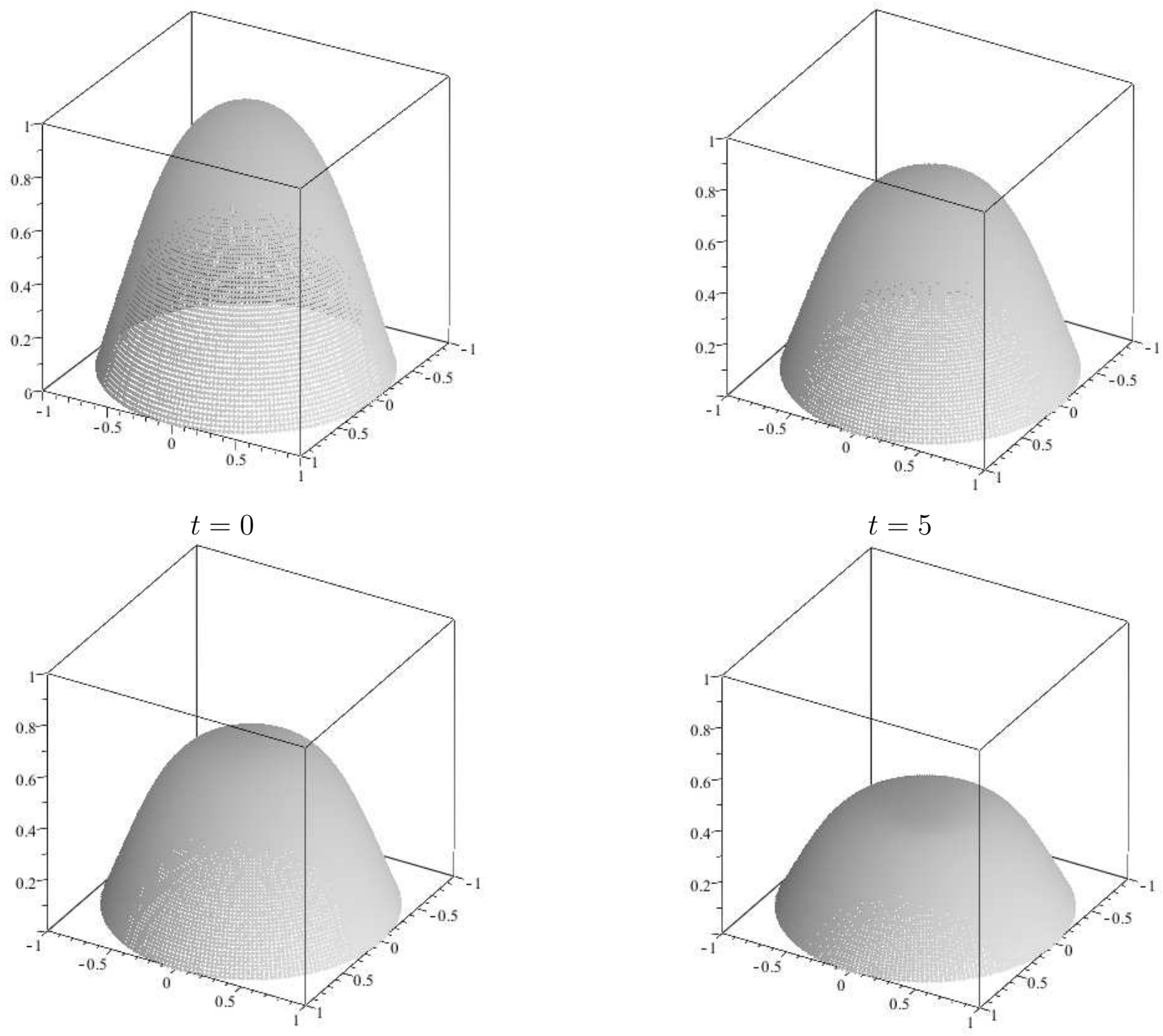

$t=10$

$t=50$

Fig. 1. The electric field potential at various time points

3. Zagrebina S.A., Sagadeeva M.A. [The Generalized Showalter - Sidorov Problem for the Sobolev Type Equations with strongly $(L, p)$-radial operator] Vestnik MaGU. Matematika. - [Bulletin of the Magnitogorsk State University. Mathematics], 2006, no 9, pp. 17-27. (in Russian)

4. Zamyshlyaeva A.A. The Phase Space of a High Order Sobolev Type Equation. The Bulletin of Irkutsk State University. Series "Mathematics", 2011, no. 4, pp. 45-57. (in Russian)

5. Sviridyuk G.A., Manakova N.A. The Dynamical Models of Sobolev Type with Showalter - Sidorov Condition and Additive "Noise". Bulletin of the South Ural State University. Series "Mathematical Modelling, Programming \& Computer Software", 2014, vol. 7, no. 1, pp. 90-103. doi: 10.14529/mmp140108 (in Russian) 
6. Sviridyuk G.A., Zagrebina S.A. Verigin's Problem for Linear Equations of the Sobolev Type with Relatively p-Sectorial Operators. Differential Equations, 2002, vol. 38, no. 12 , pp. 1745-1752.

7. Sviridyuk G.A., Keller A.V. Invariant spaces and dichotomies of solutions of a class of linear equations of the Sobolev type Izv. Vyssh. Uchebn. Zaved. Mat., 1997, no. 5, pp. 60-68. (in Russian)

8. Bogatyreva E.A., Semenova I.N. On the Uniqueness of a Nonlocal Solution In The Barenblatt - Gilman Model. Bulletin of the South Ural State University. Series "Mathematical Modelling, Programming \& Computer Software", 2014, vol. 7, no 4, pp. 113-119. doi: 10.14529/mmp140310 (in Russian)

9. Sveshnikov A.G., Al'shin A.B., Korpusov M.O. [The Nonlinear Functional Analysis and Its Applications to Partial Differential Equations]. Moscow, Nauchnyi mir Publ., 2008. (in Russian)

Ekaterina A. Bogatyreva, Postgraduate student, Department of Equation of Mathematical Physics, South Ural State University, Chelyabinsk, Russian Federation, bogatyreva-ea@susu.ac.ru.

Received February 24, 2015 